\title{
Análise de fatores pré e per-operatórios determinantes do resultado cirúrgico da tetralogia de Fallot
}

Bayard GONTIJO FILHO**, Fernando Antônio FANTINI**, João Alfredo de Paula e SILVA**, Juscelino Teixeira BARBOSA ${ }^{\star \star}$, Mário Oswaldo VRANDECIC**, Eurival Soares BRAGA*, Maria da Glória Horta MAS$\mathrm{Cl}^{\star *}$, Teresa Lúcia de Melo MASCl**, Roberto José de Alvarenga FREIRE**

RBCCV $44205-29$

\begin{abstract}
GONTIJO FILHO, B.; FANTINI, F. A.; SILVA, J. A. P.; BARBOSA, J. T.; VRANDECIC, M. O.; BRAGA, E. S.; MASCI, M. G. H.; MASCI, T. L. M.; FREIRE, R. J. A. - Análise de fatores pré e per-operatórios determinantes do resultado cirúrgico da tetralogia de Fallot. Rev. Bras. Cir. Cardiovasc., 2 (2): 102-108, 1987.

RESUMO: São analisados os resultados pós-operatórios imediatos de 98 pacientes submetidos a tratamento cirúrgico da tetralogia de Fallot (TF) num total de 109 procedimentos (29 cirurgias paliativas e 80 correções definitivas). No grupo de cirurgias paliativas, ocorreram 2 óbitos no inicio de nossa experiência, nāo havendo mortalidade nos últimos 20 pacientes operados. A técnica de Blalock-Taussig clássica foi a mais utilizada (19 casos). No grupo de correção total, houve 9 óbitos $(11,2 \%)$, sendo 7 deles no período de 1979 a 1983, ocorrendo apenas 2 óbitos nos últimos 42 casos operados. A presença de anastomose sistêmico-pulmonar prévia ( 8 casos) não interferiu na mortalidade pós-operatória. A análise retrospectiva desta experiência mostrou que os fatores que influenciaram negativamente o resultado cirúrgico foram: o baixo peso, a baixa idade, o alargamento de via de saída e alguns tipos de malformaçōes associadas. A nossa conduta atual, baseada em uma sistematização colocada em prática a partir de 1984, prevê correçāo cirúrgica em 2 estágios, com tratamento paliativo antes dos 2 anos de idade. A mortalidade combinada observada nos últimos 2 anos foi de $4,7 \%$.
\end{abstract}

DESCRITORES: tetralogia de Fallot, cirurgia, fatores de risco.

\section{INTRODUÇÃO}

A história natural da tetralogia de Fallot (TF) foi significativamente modificada com a introdução do tratamento cirúrgico, a partir de 1945 , com a descrição da anastomose subclávia-pulmonar por BLALOCK \& TAUSSIG ${ }^{4}$, seguida pela primeira correção intracardiaca realizada por LILLEHEY et alii, em $1955^{18}$.

Atualmente, os resultados obtidos, tanto no pós-operatório imediato, quanto a longo prazo, sẫo bastante satisfatórios. Apesar disso, a perspectiva de cura e de uma vida normal desses pacientes nos conduz à procura de melhores resultados.

A identificação e controle das principais variáveis que influenciam a evolução pós-operatória sāo fundamentais para este objetivo. Diversos fatores, tais como: idade ideal para correção, peso corporal, correção em 1 ou 2 tempos e regurgitaçāo pulmonar residual permanecem controvertidos.

Em 1984, introduzimos um protocolo de tratamento de TF, baseado na nossa experiência inicial (1979-1983), prevendo o tratamento paliativo (clínico e cirúrgico) e

Trabalho realizado na Santa Casa de Misericórdia de Belo Horizonte e Biocor Hospital de Doenças Lardiovasculares. Belo Horizonte, MG, Brasil. Apresentado ao 14: Congresso Nacional de Cirurgia Cardiaca, Salvador, BA, 27 e 28 de março, 1987.

- Da Santa Casa de Misericórdia de Belo Horizonte.

- Da Santa Casa de Misericórdia de Belo Horizonte e do Biocor Hospital de Doenças Cardiovasculares.

Endereço para separatas: Bayard Gontijo Filho, Praça Hugo Werneck, s/n̊, 5: andar C, Santa Efigênia. 30.000. Belo Horizonte, MG, Brasil. 
GONTIJO FILHO. B.: FANTINI. F. A.: SILVA. J. A. P.: BARBOSA. J. T.: VRANDECIC. M. O.: BRAGA. E. S.: MASCI. M. G. H.: MASCI, T. L. M.: FREIRE, R. J. A. - Análise de fatores pré e per-operatorios determinantes do resultado cirúrgico da tetralogia de Fallot. Rev. Bras. Cir. Cardiovasc. 2 (2): 102-108, 1987.

a correçāo cirúrgica em 2 estágios. O presente trabalho visa a analisar o resultado obtido nestas duas etapas de tratamento e identificar as principais variáveis que afetaram significativamente o pós-operatório destes pacientes.

\section{MATERIAL}

No período de 1979 a 1986 , foram operados 98 pacientes portadores de TF, sendo realizadas 29 cirurgias paliativas e 80 correções definitivas (total de 109 procedimentos). Todos os pacientes foram submetidos a estudo angiocardiográfico, que foi a principal base do diagnóstico anatômico pré-operatório.

No grupo de cirurgias paliativas, a idade variou de 2 dias a 2,7 anos, estando $85 \%$ no 1 : ano de vida.

A técnica clássica de Blalock-Taussig (BT) foi empregada 19 vezes $(65,5 \%)$ sendo as demais realizadas com interposição de prótese de PTFE (34,5\%). Até 1984 , o BT clássico foi realizado em todas as crianças; posteriormente, o tratamento paliativo foi alterado, incluindo o uso de betabloqueadores e a anastomose de BT modificada (PTFE), em crianças menores de 6 meses de vida. Oito crianças deste grupo foram, posteriormente, submetidas a correção definitiva.

A correção total foi efetuada em 80 pacientes, cuja idade variou de 1 ano a 35 anos; 13 pacientes encontravam-se com idade superior a 18 anos $(16,2 \%)$, enquanto que 13 crianças foram operadas em idade inferior de 2 anos (16,2\%). Com relação ao peso corporal, 17 crianças apresentavam peso inferior a $10 \mathrm{Kg}(21,7 \%)$.

Dos 80 pacientes submetidos a correção total, 8 deles apresentavam uma anastomose sistêmico-pulmonar prévia, que foi ligada no ato operatório. As principais malformações associadas à TF encontradas neste grupo estão relacionadas no Quadro 1. Não foram incluídos os pacientes portadores de atresia pulmonar e nem aqueles portadores de comunicação interatrial.

\section{QUADRO 1}

MALFORMAÇÕES ASSOCIADAS A TF ENCONTRADAS EM 80 PACIENTES SUBMETIDOS A CORREÇATO TOTAL

\begin{tabular}{|c|c|}
\hline & INCIDENCIA \\
\hline CIV subpulmonar ............................ & 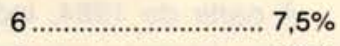 \\
\hline Agenesia de valva pulmonar ....... & $5 \ldots \ldots \ldots \ldots \ldots \ldots \ldots \ldots \ldots \ldots, 6,2 \%$ \\
\hline Agenesia de ramo de AP .............. & 3 \\
\hline Drenagem ven. sist. anômala ..... & 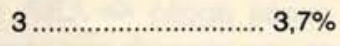 \\
\hline Artéria coronária anômala ........... & 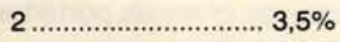 \\
\hline Drenagem anômala total VP ........ & 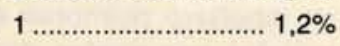 \\
\hline
\end{tabular}

Total

20

$25,0 \%$
A técnica cirúrgica empregada para correção total foi praticamente a mesma durante todo o periodo de observação e consistiu em esternotomia mediana, com retirada do timo e abertura do pericárdio. A anastomose sistêmico-pulmonar, quando presente, foi dissecada e isolada antes do inicio da circulação extracorpórea (CEC). Após heparinizaçāo (3 $\mathrm{mg} \mathrm{Kg}$ ), procedeu-se à canulação de aorta ascendente e de ambas as cavas. Após estabelecimento de parada cardiaca cardioplégica, reparamos as bordas do VD e realizamos ventriculotomia direita longitudinal. Em seguida, procedemos à ressecção de musculatura infundibular e realizamos a comissurotomia pulmonar, quando indicada. Medimos o anel pulmonar com vela de Hegar, decidindo-se pela ampliaçāo, ou não, de via de saída do ventrículo direito (VSVD). A comunicação interventricular foi fechada sempre com remendo de Dacron e fixada, na maioria dos casos, com pontos separados de Prolene $4.0 \mathrm{com}$ almofadas.

Em todos os pacientes, usamos um retalho de Dacron, ou pericárdio bovino, para fechamento da ventriculotomia, mesmo quando se procedeu ao alargamento da VSVD. Quando se tornou necessário este alargamento, confeccionamos, cuidadosamente, o remendo, procurando evitar uma dilatação excessiva do anel pulmonar. Não usamos mecanismos valvulares na VSVD em nenhum paciente, mesmo naqueles com síndrome de agenesia de valva pulmonar, ou naqueles portadores de agenesia de um ramo aa artéria pulmonar.

Todos os estudos hemodinâmicos pré-operatórios foram revistos, sendo realizadas medidas do diâmetro da artéria pulmonar e da aorta ascendente (acima do plano valvar), estabelecendo-se a relaçăo diâmetro $\mathrm{Ap} /$ Ao.

\section{RESULTADOS}

No grupo de cirurgias paliativas (26 pacientes), a mortalidade pós-operatória foi de 2 crianças $(7,6)$, tendo ambas ocorrido no início da nossa experiência. A primeira foi conseqüente a distúrbio metabólico grave préoperatório e a outra, devido a paralisia diafragmática e insuficiência respiratória. Três anastomoses de BT clássico realizadas em crianças com menos de 6 meses de idade se mostraram insuficientes no pós-operatório, sendo necessária nova cirurgia paliativa. Não houve mortalidade dos pacientes submetidos a anastomose com PTFE. O Quadro 2 resume os dados referentes às cirurgias paliativas.

Betabloqueadores foram empregados em 16 crianças com crises hipóxicas, estando 12 delas no primeiro ano de vida. Destas 12 crianças, 8 foram submetidas a anastomoses sistêmico-pulmonares devido a recidiva da crise. A droga demonstrou-se efetiva, basicamente nos primeiros meses de vida, e as principais complicações encontradas foram broncoespasmo e hipersecreção pulmonar. 
GONTIJO FILHO, B.: FANTINI, F. A.: SILVA, J. A. P.: BARBOSA. J. T.: VRANDECIC, M. O.: BRAGA. E. S.: MASCI. M. G. H.: MASCI, T. L. M.: FREIRE, R. J. A. - Analise de fatores pre e per-operatorios determinantes do resultado cirurgico da tetralogia de Fallot. Rev. Bras. Cir. Cardiovasc. 2 (2): 102-108. 1987

QUADRO 2

RESULTADO PÓS-OPERATÓRIO DOS PACIENTES SUBMETIDOS A ANASTOMOSE SISTEMICO-PULMONAR (29 PROCEDIMENTOS)

\begin{tabular}{|c|c|c|c|c|}
\hline \multicolumn{3}{|c|}{$\begin{array}{c}\text { ANASTOMOSE DE BT } \\
\text { CLASSICA }\end{array}$} & \multicolumn{2}{|c|}{$\begin{array}{l}\text { ANASTOMOSE DE BI } \\
\text { MODIFICADA (PTFE) }\end{array}$} \\
\hline PERIODO & $79-83$ & $84-86$ & 79.83 & $84-86$ \\
\hline N: Casos & 15 & 4 & 0 & 10 \\
\hline Oclusão & 3 & 0 & 0 & 0 \\
\hline Óbitos & 2 & 0 & 0 & 0 \\
\hline
\end{tabular}

No grupo de cirurgia corretiva ( 80 pacientes), ocorreram 7 óbitos, nos primeiros 38 casos operados $(18,2 \%)$ e apenas 2 nos últimos 42 pacientes $(4,7 \%)$. A análise deste resultado relacionado a faixa etária, peso, relação de diâmetro Ap/Ao, presença de alargamento transanular e presença de anastomose SP prévia estão sintetizados no Quadro 3. Contribuíram, efetivamente, para maior mortalidade a idade inferior a 2 anos, o peso inferior a $10 \mathrm{Kg}$ e a presença de alargamento transanular da VSVD.

QUADRO 3

FATORES INFLUENTES NA MORTALIDADE PÓS-OPERATÓRIA DA CORRECAOA TOTAL DA TF (80 CASOS)

\begin{tabular}{|c|c|c|c|}
\hline & VARIAVEEIS & $\begin{array}{c}N^{\circ} \\
\text { CASO }\end{array}$ & ORTALIDADE \\
\hline \multirow{2}{*}{ Idade } & Abaixo de 2 anos & 13 & $6(46,5 \%)$ \\
\hline & Acima de 2 anos & 67 & $3(4,4 \%)$ \\
\hline \multirow{2}{*}{ Peso } & Abaixo de $10 \mathrm{Kg}$ & 17 & $5(29,4 \%)$ \\
\hline & Acima de $10 \mathrm{Kg}$ & 63 & $4(6,3 \%)$ \\
\hline Relação & Menor de 0,3 & 2 & $1(50,0 \%)$ \\
\hline Diâmetro & Entre 0,3 e 0,5 & 27 & $3(11,1 \%)$ \\
\hline $\mathrm{Ap} / \mathrm{Ao}$ & Maior de 0.5 & 51 & $5(9,8 \%)$ \\
\hline \multirow{2}{*}{$\begin{array}{l}\text { Presença de } \\
\text { Anastomose SP }\end{array}$} & Sim & 8 & 0 \\
\hline & Não & 72 & $9(12,5 \%)$ \\
\hline \multirow{2}{*}{$\begin{array}{l}\text { Alargamento } \\
\text { de VSVD }\end{array}$} & Sim & 51 & $8(15,6 \%)$ \\
\hline & Não & 29 & $1(3,4 \%)$ \\
\hline
\end{tabular}

Quanto às malformações associadas, a presença de comunicação interventricular (CIV) subpulmonar, a drenagem venosa sistêmica anômala e a síndrome de agenesia de valva pulmonar não influenciaram a mortali- dade pós-operatória. Entre os 3 pacientes portadores de agenesia de ramo de artéria pulmonar, houve um óbito pós-operatório. Faleceram, ainda, 1 criança portadora de drenagem anômala total de veias pulmonares (DATVP) do tipo intracardiaco e outra com artéria coronária anômala.

\section{DISCUSSÃO}

Estudos recentes demonstram a excelente evoluçāo pós-operatória a longo prazo, dos pacientes submetidos a correção total de TF. ZHAO et alii ${ }^{29}$ relatam $85 \%$ de sobrevida após 16 anos de cirurgia, enquanto que KIRKLIN ${ }^{15}$ encontrou resultados ainda superiores, observando $93 \%$ de sobrevida após 10 anos de cirurgia e $91 \%$ após 20 anos. Além disso, a grande maioria desses pacientes apresenta ótima capacidade funcional, estando muito poucos com restriçōes da atividade física, ou em uso de drogas ${ }^{15}$. 26.

Ainda assim, o resultado global do tratamento cirúrgico da TF pode ser melhorado, uma vez que fatores, tais como a mortalidade, pós-operatório imediato, as reoperaçōes tardias, as mortes súbitas por arritmias e a disfunção ventricular direita necessitam, maiores estudos e melhor controle por parte do cirurgião.

Procuramos, nesta primeira parte do nosso trabalho, avaliar os principais fatores que influenciaram a evolução pós-operatória imediata dos pacientes portadores de TF operados no nosso Serviço. O início da nossa experiência na correção cirúrgica da TF foi acompanhado de um indice relativamente alto de mortalidade $(17,9 \%)$. Por esse motivo, introduzimos, a partir de 1984 , um protocolo de tratamento da TF, levando em consideração os principais fatores que influenciaram este resultado inicial. A análise retrospectiva dessa experiência e a discussāo dos diversos fatores influentes na mortalidade pós-operatória tornaram-se fundamentais para a obtenção de melhores resultados e para a elaboração da nossa conduta atual.

Cirurgias Paliativas - As anastomoses sistêmico pulmonares (ASP) sempre foram parte importante da nossa abordagem na TF. No início de nossa experiência, indicávamos as ASP principalmente para as crianças no primeiro ano de vida e para aquelas que apresentavam má anatomia no estudo hemodinâmico.

A partir de 1984, indicamos cirurgia paliativa em todas as crianças menores de 2 anos de idade, que não respondiam bem ao tratamento clínico. Inicialmente, a nossa opção de ASP, não só para a TF, mas para todas as crianças portadoras de cardiopatias congênitas com hipofluxo pulmonar era a técnica de BT clássica, baseados nos relatos da literatura, que preconizavam seu uso mesmo em recém-nascidos ${ }^{8 .}{ }^{17}, 20$. Posteriormente, observamos alguns casos de oclusão, ou hipoflu- 
GONTIJO FILHO. B.: FANTINI. F. A.: SILVA, J. A. P.: BARBOSA. J. T.: VRANDECIC. M. O.: BRAGA. E. S.: MASCI. M. G. H.: MASCI, T. L. M.: FREIRE. R. J. A. - Análise de fatores pré e per-operatorios determinantes do resultado cirurgico da tetralogia de Fallot. Rev. Bras. Cir. Cardiovasc. 2 (2): 102-108. 1987.

xo, de anastomoses realizadas em crianças menores de 6 meses de idade, o que nos levou à utilização cada vez mais freqüente da técnica de BT modificado, conforme preconizado por DeLEVAL et alii ${ }^{9}$. Esta técnica foi introduzida, como rotina, no nosso Serviço, para crianças com menos de 6 meses de idade, a partir de 1984 , observando-se melhoria importante do resultado. Para as crianças acima de 6 meses de idade, continuamos preferindo o BT clássico, já que este tem apresentado bom desempenho. Nos pacientes submetidos a correção total com ASP prévia (8 casos), a ligadura de ASP não apresentou dificuldades e nāo interferiu no resultado pósoperatório. Este dado, bem como a ausência de mortalidade nas últimas 20 crianças submetidas a ASP são importantes, na nossa opção de correção cirúrgica em 2 tempos. São também relevantes as observaçōes que comprovam que a presença de uma ASP prévia à correção total não é um fator agravante, na evolução a longo prazo destes pacientes ${ }^{2,26,29}$.

Drogas Betabloqueadoras - O uso de betabloqueadores não é aceito por todos como forma de paliação da TF. A nossa experiência em 16 crianças submetidas a este tratamento, com uma dose variável de $1-4 \mathrm{mg} / \mathrm{Kg}$ / dia, embora pequena, apresentou alguns dados importantes. A droga se mostrou efetiva, principalmente nos 6 primeiros meses de vida, havendo, a partir de então, um número relativamente alto de insucessos, não sendo, portanto, eficaz isoladamente, para levar uma criança sintomática nos primeiros meses de vida até a idade de correção total. Alguns efeitos colaterais, como a hipersecreção pulmonar, o broncoespasmo e a bradicardia, foram importantes na determinação da dose de manutenção e, algumas vezes, forçaram a suspensão da mesma. Não indicamos o betabloqueador como preventivo de crises hipóxicas e, atualmente, seu uso está praticamente restrito às crianças sintomáticas com menos de 6 meses de idade. Nas crianças entre 6 meses e 2 anos de idade, com crises de hipóxia, ou naquelas não controladas pelo betabloqueador, optamos pela ASP.

Idade e Peso para Correção Total - Observamos uma incidência maior de óbitos em crianças menores de 2 anos de idade e com peso corporal inferior a 10 $\mathrm{Kg}$. Este fato, apesar de identificado também por outros autores, como importantes no resultado cirúrgico', não é considerado, em alguns Serviços que apresentam excelentes resultados de correção total durante o primeiro ano de vida ${ }^{6}{ }^{28}$. Devido à mortalidade alta em crianças abaixo de 2 anos, na nossa experiência, indicamos a correção total entre os 2 e 4 anos de idade. $O$ baixo peso corporal, apesar de aumentar o risco cirúrgico $(29,4 \%)$, não foi tão importante quanto à idade, nos nossos casos. A idade adulta não foi fator agravante do resultado cirúrgico, conforme já observado em outras experiências ${ }^{7}$. Estes casos foram acompanhados de um número significativamente maior de reoperaçōes para revisão de hemostasia ( 5 reoperaçōes em 13 pacientes).
Relação Diâmetro Ap Ao - OKU et alii ${ }^{22}$ demonstraram um pior resultado cirúrgico em pacientes portadores de TF, com relação aos diâmetros do tronco da artéria pulmonar e da aorta ascendente inferior a 0,3. Levantamos estes dados nos nossos pacientes, porém o número de casos com índice inferior a 0,3 foi muito pequeno, não permitindo conclusões definitivas a este respeito. Confirmamos, por outro lado, a observação de que o resultado pós-operatório não foi significativamente diferente entre os pacientes com índices situados na faixa de 0,3 a 0,5 e aqueles com indices superiores a 0,5 .

Alargamento de Via de Saida de VD - O alargamento da VSVD foi realizado segundo os critérios preconizados por PACÍFICO et alii ${ }^{23}$, em 51 pacientes da nossa série $(63,7 \%)$ e alterou de forma significativa o resultado pós-operatório. Este alto índice de alargamentos transanulares na nossa série coincide com o observado em outras séries cirúrgicas ${ }^{2}$. 7. 11. Observamos $15,6 \%$ de mortalidade nos pacientes submetidos ao alargamento da VSVD, contra apenas $3,4 \%$ do outro grupo. Este pior resultado imediato dos pacientes submetidos a esta técnica, bem como observaçōes no pós-operatório tardio que sugerem um efeito deletério da insuficiência pulmonar crônica sobre a capacidade funcional do $V^{5}$. têm proporcionado modificações técnicas durante o ato cirúrgico.

Ultimamente, alguns autores têm preconizado o uso de monocúspides suturadas no retalho do alargamento de VSVD, alegando melhor evolução pós-operatória ${ }^{13}$. Outros relatos contestam a eficácia desta técnica ${ }^{27} \mathrm{e}$, além disto, faltam maiores observaçōes a longo prazo para uma aferição definitiva a respeito da validade do uso das monocúspides. $\mathrm{O}$ uso de prótese valvular na VSVD não se justifica, como opção primária na correção da TF, devido ao alto índice de complicaçōes destas próteses, principalmente em crianças ${ }^{3}$. A sua utilizaçāo está reservada aos casos que apresentam insuficiência cardiaca pós-operatória refratária associada à regurgitação pulmonar.

Outra tentativa de se reduzir o número de alargamentos de VSVD consiste na aceitação de maior pressão sistólica na cavidade ventricular direita durante o ato cirúrgico. KIRKLIN ${ }^{15}$ tem aceito um indice trans-operatório de 0,85 da relação da pressão sistólica VD/VE baseado em observaçōes que demonstram uma queda significativa deste indice, 24 horas após a cirurgia, e também ao longo dos anos ${ }^{10.21}$.

Por outro lado, encontramos, na literatura, opiniōes que minimizam o papel da regurgitação pulmonar crônica sobre o VD7. ${ }^{24}$. Desta forma, a discussāo sobre alargamento de VSVD permanece em aberto e mais observaçōes a longo prazo se fazem necessárias para uma conduta segura nos pacientes portadores de TF com má anatomia. Nossa conduta atual é de evitar a ampliação do VSVD em crianças menores de 2 anos de idade, 
GONTIJO FILHO, B.: FANTINI, F. A.: SILVA. J. A. P.: BARBOSA. J. T.: VRANDECIC. M. O.: BRAGA. E. S.: MASCI. M. G H.: MASCI, T. L. M.: FREIRE, R. J. A. - Análise de fatores pré e per-operatorios determinantes do resultado cirurgico da tetralogia de Fallot. Rev. Bras. Cir. Cardiovasc. 2 (2): 102-108. 1987

porém nāo nos preocupamos em criar mecanismos valvulares, quando a empregamos.

Malformaçōes Associadas - A presença de outras anomalias foi um fator agravante, na nossa experiência, tendo ocorrido 3 óbitos em 19 pacientes com malformações associadas. A comunicação interventricular do tipo subpulmonar, ou subarterial, não alterou o prognóstico pós-operatório. Apenas 1 dos pacientes apresenta CIV residual moderada e deverá ser submetido a reoperaçāo. A ocorrência de drenagem venosa anômala total de veias pulmonares (DATVP), que é bastante rara na TF, foi encontrada em 1 caso. Este paciente apresentava um DATVP do tipo intracardiaco e faleceu em baixo débito, no pós-operatório. A presença de veias pulmonares relativamente finas, concorrendo para uma possivel hipertensāo pulmonar pós-operatória associada ao alargamento da VSVD, deve ter sido o fator determinante do mau resultado.

Com relação à síndrome de agenesia da valva pulmonare agenesia de um ramo da $\mathrm{AP}$, as nossas observaçōes e os dados da literatura suscitam maior discussāo, uma vez que existe controvérsia com relação ao uso primário de prótese valvular na VSVD, nestes pacientes $^{12}, 14.19 .25$. Os 5 pacientes operados por nós, com SAVP (todos acima de 3 anos de idade), foram corrigidos sem uso de próteses e apresentaram boa evolução. Em 3 deles, foi realizado estudo hemodinâmico pós-operatório, que revelou bom resultado, o que nos leva a acreditar ser indispensável o implante valvular como primeira opção, nestes casos.

Nos pacientes com ARAP, observamos 1 óbito em paciente adulto, com agenesia de ramo direito de AP e com uma relação Dap/Ao muito baixa $(0,22)$. $O$ implante de prótese, neste caso, talvez pudesse concorrer para um melhor resultado. Por outro lado, tivemos uma criança de
3 anos de idade, portadora de agenesia do ramo esquerdo de AP e hipoplasia de VSVD, na qual planejamos o uso de prótese valvular para sua correção. Durante a cirurgia, entretanto, foi encontrada uma artéria direita anômala cruzando a região infundibular. Esta coronária foi dissecada e amplamente mobilizada, sendo optado apenas pelo alargamento sob a mesma. A evolução pósoperatória foi excelente. Desta forma, achamos que os casos de ARAP devem ser analisados individualmente, levando-se em consideração o desenvolvimento da artèria pulmonar presente, decidindo-se, então, pela colocação, ou não, de prótese, durante a cirurgia inicial.

\section{CONCLUSŌES}

1) A mortalidade pós-operatória foi substancialmente reduzida, na nossa experiência, através da introdução de uma sistematização que prevê a correçāo em 2 tempos, com tratamento paliativo antes dos 2 anos de idade.

2) As drogas betabloqueadoras mostraram-se eficazes como tratamento paliativo, nas crianças até 6 meses de idade.

3) A anastomose de BT clássica apresentou indice elevado de complicaçōes, nos primeiros 6 meses de vida. A anastomose com prótese de PTFE foi superior, nesta faixa etária.

4) Os fatores que determinaram maior mortalidade pós-operatória, na nossa experiência, foram: idade inferior a 2 anos, peso corporal inferior a $10 \mathrm{~kg}$, alargamento do VSVD e presença de malformaçōes associadas.

5) Não constituíram fatores agravantes na correção total: a presença de ASP prévia, a idade adulta e a relação de diâmetro $\mathrm{Ap} / \mathrm{Ao}$ acima de 0,3 .

RBCCV $44205-29$

GONTIJO FILHO, B.; FANTINI, F. A.; SILVA, J. A. P.; BARBOSA, J. T.; VRANDECIC, M. O.; BRAGA, E. S.; MASCI, M. G. H.; MASCI, T. L. M.; FREIRE, R. J. A. - Analysis of the factors pre and post-operatory. as determinants of the surgical results of the tetralogy of Fallot. Rev. Bras. Cir. Cardiovasc., 2 (2): 102-108, 1987.

ABSTRACT: We report the immediate postoperative results of 98 patients who underwent surgical treatment for tetralogy of Fallot. A paliative operation was employed in 29 children and total correction was performed in 80 patients (109 operations). There were 2 postoperative deaths in the group of paliative treatment which occurred in the beggining of our experience and no deaths were found in the last 20 operations. In the group of patients who underwent total correction there were 9 deaths $(11,2 \%)$, but only 2 of these deaths occurred in the last 42 patients operated. The presence of a previous shunt ( 8 patients) did not interfere with the postoperative result. The factors that determined a higher postoperative mortality were: age under 2 years, weight under $10 \mathrm{~kg}$, presence of transannular patch of the right ventricle and some kinds of associated anomalies. Our combined operative mortality of $4.7 \%$ in the last 2.5 years, is the support of our current choice for 2-stage surgical correction.

DESCRIPTORS: tetralogy of Fallot, surgery, risk factors. 
GONTIJO FILHO, B.: FANTINI, F. A.: SILVA. J. A. P.: BARBOSA, J. T.: VRANDECIC. M. O.: BRAGA. E. S.: MASCI. M. G H.; MASCI. T. L. M.: FREIRE, R. J. A. - Análise de fatores pré e per-operatorios determinantes do resultado cirurgico da tetralogia de Fallot. Rev. Bras. Cir. Cardiovasc. 2 (2): 102-108. 1987

\section{REFERÊNCIAS BIBLIOGRÁFICAS}

1 ARCINIEGAS, E.; FAROOKI, Z. Q.: HAKIMI, M.; GREEN. E. W. - Results of two-stage surgical treatment of tetralogy of Fallot. J. Thorac. Cardiovasc. Surg.. 79 (6): $876-883,1980$.

2 ARCINIEGAS, E.: FAROOKI, Z. Q.: HAKIMI, M.: PERRY. B. L.; GREEN, E. W. - Early and late results of total correction of tetralogy of Fallot. J. Thorac. Cardiovasc. Surg., 80 (5): 770-778, 1980.

3 BISSET III, G. S.; SCHWARTZ, D. C.; BENZING III, G.: HELMSWORTH, J; SCHREIBER, J. T.; KAPLAN, S. - Late results of reconstruction of the right ventricular outflow tract with porcine xenografts in children. Ann. Thorac. Surg., 31 (5): 437-443, 1981.

4 BLALOCK, A. \& TAUSSIG, H. B. - The surgical treatment of malformations of the heart: in which there is pulmonary stenosis or pulmonary atresia. JAMA, 128 (3): 189-202, 1945.

5 BOVE, E. L.; BYRUM, C. J.; THOMAS, F. D.; KAVEY R. W.; SONDHEIMER, H. M.; BLACKMAN, M. S.; PARKER, Jr., F. B. - The influence of pulmonary insufficiency on ventricular function following repair of Tetralogy of Fallot: evaluation using Radionuclide ventriculography. J. Thorac. Cardiovasc. Surg., 85 (5): 691-696. 1983.

6 CAStañeda, A. R.; FREed, M. D.; WILliams, R. G.: NORWOOD, W. I. - Repair of tetralogy of Fallot in infancy: early and late results. J. Thorac. Cardiovasc. Surg., 74 (3): 372-381, 1977.

7 CHIARELLO, L.; MEYER, J.; WUKASCH, D. C.; HALLMAN, G. L.: COOLEY, D. A. - Intracardiac repair of tetralogy of Fallot: five-year review of 403 patients. J. Thorac. Cardiovasc. Surg., 70 (1): 529-535, 1976.

8 CHOPRA, P. S.; LEVY, J. M.; DOCUMOS, G. C.; BERKOTT, H. A.; LORING, L. L.; KHAN, D. R. - The Blalock-Taussig operation: the procedure of choice in the hypoxic infant with tetralogy of Fallot. An Thorac. Surg., 22 (3): 235-238, 1976.

9 DeLEVAL, M. R.; Mc KAY, R.; JONES, M.; STARK, J.; MacCARTNEY, F. J. - Modified Blalock-Taussig shunt: use of subclavian artery orifice as flow regulator in prosthetic systemic-pulmonary artery shunts. J. Thorac. Cardiovasc. Surg., 81 (1): 112-119, 1981.

10 GOOR, D. A.; SMOLINSKY, A.; MOHR, R.; CASPI, J.; SHEM-TOV, A. - The drop of residual right ventricular pressure 24 hours after conservative infundibulectomy in repair of tetralogy of Fallot. J. Thorac. Cardiovasc. Surg., 81 (6): 897-905, 1981.

11 HAMMON Jr., J. W.; HENRY Jr., C. L.; MERRILL, W. H.; GRAHAM Jr., T. P.; BENDER Jr., H. W. - Tetralogy of Fallot: selective surgical management can minimize operative mortality. Ann. Thorac. Surg., 40 (2): 280-284, 1985.
12 ILBAWI, M. N.: IDRISS, F. S.: MUSTER. A. J.: WESSEL, H. V.: PAUL, M. H.: DeLEON. S. Y. - Tetralogy of Fallot with absent pulmonary valve. Should valve insertion be part of the intra cardiac repair? J. Thorac. Cardiovasc. Surg., 81 (6): 906-915, 1981.

13 IONESCU. M. I.: TANDON. A. P.: MacCARTNEY, F. J. Long-term sequential hemodynamic evaluation of right ventricular outflow tract reconstruction using a valve mechanism. Ann. Thorac. Surg.. 27 (4): 426-434, 1979

14 JUCÁ. E. R.: CARVALHO Jr., W.: OLIVEIRA. F. M.: ARAÚJO, J. A. R.: MONT ALVERNE, R.: PAES Jr, J. N.: SOUZA, J. R. - Tratamento da tetralogia de Fallot com agenesia da artéria pulmonar esquerda: relato de 2 casos. Arq. Bras. Card., 38 (1): 39-43, 1982.

15 KIRKLIN, J. W. \& BARRAT-BOYES, B. G. - Cardiac Surgery. New York, John Wiley \& Sons, 1986.

16 KIRKLIN, J. W.; BLACKSTONE, E. H.; KIRKLIN, J. K. PACIFICO, A. D.: ARAMENDI, J.; BARGERON Jr., L. M. - Surgical results and protocols in the spectrum of tetralogy of Fallot. Ann. Thorac. Surg., 198 (3): 251-265, 1981

17 LAKS, H.; FAGAN, L.; BARNER, H. B.: WILLMAN, V. L. - The Blalock-Taussing shunt in the neonate. Ann. Thorac. Surg., 25 (3): 220-224, 1978.

18 LILLEHEI, C. W.; COHEN, M.; WARDEN, H. E.; READ R. C.: AUST, J. B.; DeWALL, R. A.; VARCO, R. L. - Direct vision intracardiac surgical correction of the tetralogy of Fallot, pentalogy of Fallot and pulmonary atresia defects. Ann. Thorac. Surg., 142: 318-445, 1955.

19 McCAUGhAN, B. C.; DANIELSON, G. K.; DRISCOLL, D. S.; McGOON, D. C. - Tetralogy of Fallot with absent pulmonary valve. J. Thorac. Cardiovasc. Surg., 89 (2): 280-287, 1985.

20 MARBARGER, J.; SANDZA, H.; HARTMANN, A.; WELDON, C. - Blalock-Taussing anastomosis preferred shunt in infants and newborns. Circulation, 58 (Supl. 1): $73-77,1978$.

21 MURAOKA, R.; YOKOTA, M.; MATSUDA, K.; TABOTA, R.; HIKASA, Y. - Long-term hemodynamic evolution of primary total correction of tetralogy of Fallot during the first two years of life. Arch. Jpn. Chir., 42: 315-320. 1973.

22 OKU, H.; SHIROTANI, H.; YOKOLYAMA, T.; YOKOTA, Y.; KAWAI, J.; MORI, A.; KANZAKI, Y.; MAKINO, S. ANDO, F.; SETSUIE, N. - Postoperative size of the right ventricular outflow tract and optimal age in complete repair of tetralogy of Fallot - Ann. Thorac. Surg. 25 (4): 322-328, 1978

23 PACÍFICO, A. D.; KIRKLIN, J. W.; BLACKSTONE, E. H. - Surgical management of pulmonary stenosis in tetralogy of Fallot. J. Thorac. Cardiovasc. Surg., 74 (3): 382-395, 1977. 
GONTIJO FILHO, B.: FANTINI. F. A.: SILVA, J. A. P.: BARBOSA, J. T.: VRANDECIC, M. O.: BRAGA. E. S.: MASCI. M. G. H.: MASCI, T. L. M.: FREIRE, R. J. A. - Análise de fatores pré e per-operatórios determinantes do resultado cirúrgico da tetralogia de Fallot. Rev. Bras. Cir. Cardiovasc. 2 (2): 102-108, 1987.

POIRIER, R. A.: MCGOON. D. C.: DANIELSON, C. K.: WALLACE, R. B.; RITTER, D. G.; MOODIE, D. S.; WILTSE, C. G. - Late results after repair of tetralogy of Fallot. J. Thorac. Cardiovasc. Surg., 73 (2): 700-708, 1977.

PRATES, P. R.; SANTANA, J.; KALIL, R. A.; LUCCHESE. F. A.; BERTOLETTI, V. E.; NESRALLA, I. A. - Congenital absence of pulmonary valve and ventricular septal defect: surgical repair with homologous dura-mater valvular prosthesis. Bull. Texas Heart Inst., 4 (2): 222-229, 1977.

ROSENTHAL, A.; BEHRENDT, D.: SLOAN, H.; FERGUSON, P.; SNEDECOR, S. N.; SCHORK, A. - Longterm prognosis ( 15 to 26 years) after repair of tetralogy of Fallot: I. survival and symptomatic status. Ann. Thorac. Surg., 38 (2): 151-156, 1984.
27 SIEVERS, $H$. H.: LANGE, P. E.: REGENSBURGER, D. YANKAH, C. A.: ONNASCH, D. G. W.: BURSCH, J.: HEINTZEN, P. H.: BERNHARD, A. - Short-term hemodynamic results after right ventricular outflow tract reconstruction using a cusp-bearing transannular patch. J. Thorac. Cardiovasc. Surg.. 86 (5): 777-783. 1983.

28 TUCKER, W. Y.: TURLEY, K.; ULLYOT, D. J.: EBERT, P. A. - Management of symptomatic tetralogy of Fallot in the first year of life. J. Thorac. Cardiovasc. Surg. 78 (4): 494-501, 1979.

29 ZHAO, H.: MILLER, D. C.: REITZ, B. A.; SHUMWAY, N E. - Surgical repair of tetralogy of Fallot: long-term follow-up with particular emphasis on late death and reoperation. J. Thorac. Cardiovasc. Surg., 89 (2): 204-220, 1985 